\title{
Design of an Edge Slotted Waveguide Antenna Array Based on T-Shaped Cross-Section Waveguide
}

\author{
Teng Li and Wenbin Dou \\ State Key Laboratory of Millimeter Waves, Southeast University, Nanjing 210096, China \\ Correspondence should be addressed to Teng Li; liteng_nj@163.com
}

Received 22 January 2017; Revised 14 May 2017; Accepted 4 June 2017; Published 6 July 2017

Academic Editor: Muhammad Ramlee Kamarudin

Copyright ( 2017 Teng Li and Wenbin Dou. This is an open access article distributed under the Creative Commons Attribution License, which permits unrestricted use, distribution, and reproduction in any medium, provided the original work is properly cited.

\begin{abstract}
An edge slotted waveguide antenna array based on T-shaped cross-section radiating waveguide is proposed. The T-shaped waveguide is analyzed and designed to operate in dominant mode around the center frequency, which has a lower profile compared with the rectangular one. The radiating slots are etched and rotated alternatively on the broadened top plate without cutting into the adjacent walls. The metal fences are inserted between slots to reduce the mutual coupling and surface wave. Therefore, the sidelobe level in E-plane is well suppressed. A $2 \times 8$ antenna array working at Ka-band is designed and fabricated. The measured results agree well with simulations which demonstrate this novel waveguide structure.
\end{abstract}

\section{Introduction}

Slotted waveguide antenna array is a good candidate in the areas of radar and communication systems due to its attractive features, such as low power loss, low cross-polarization levels, low profile, high power capacity, high efficiency, and accurate control of amplitude and phase distributions. Some edge slot antenna arrays have been researched [1-6]. The edge slot waveguide antenna can be integrated with the other antennas for dual polarization applications [7-9]. The slots etched on the narrow wall of the waveguide are usually cut into the adjacent broad walls [1-3] or folded as Z-shaped [46] to achieve the resonance.

In this paper, a novel edge slotted waveguide antenna array is proposed based on the T-shaped cross-section waveguide. Owing to the top plate which is broadened, the radiating slots can be etched on the top without cutting into the adjacent walls or folded as Z-shaped. Furthermore, the waveguides with T-shaped cross-section can also reduce the profile compared with regular ones. The metal fences are inserted between slots to suppress mutual coupling and surface wave which are beneficial to improve the radiation performance. The effect of these fences on radiating slots is analyzed as well. A bend waveguide transition is invented to transform the waveguide from $E$-plane to $H$-plane. Finally, a
$2 \times 8$ antenna array working at $35 \mathrm{GHz}$ is designed by using full wave EM simulator and validated experimentally.

\section{The Analysis of T-Shaped Waveguide}

The rectangular waveguides are usually used as a radiating waveguide for the edge slotted waveguide antennas with the slots cutting into the broad walls for resonance. The novel T-shaped waveguide is firstly introduced as a radiating waveguide in [10] and the cross-section of the waveguide becomes T-shaped as shown in Figure 1(a). The port field in the dominant mode and the surface current on the top plate of the T-shaped waveguide are studied and they are depicted in Figure 1(b) compared with a rectangular one. It can be seen that the port field and surface current on the top plate of the two waveguides are similar to the TE dominant mode. Therefore, the inclined radiating slots etched on the top plate of the T-shaped waveguide can cut the surface current and be designed like the regular ones.

The higher order propagation mode may be introduced into the T-shaped waveguide such as $\mathrm{TE}_{01}$ mode owing to the broadened top plate. Therefore, the operating mode of the T-shaped waveguide should be analyzed and a rectangular waveguide with the same guide wavelength is proposed for comparison. 


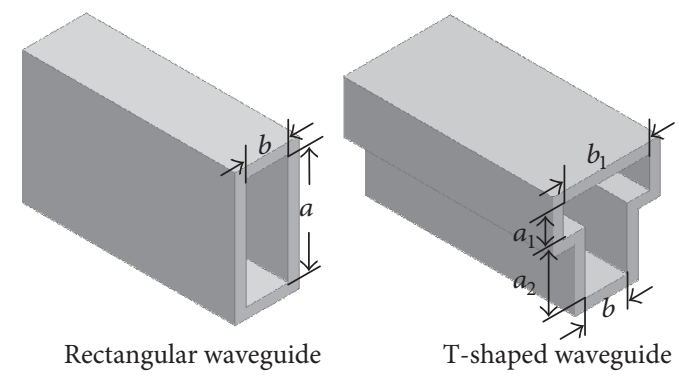

(a)

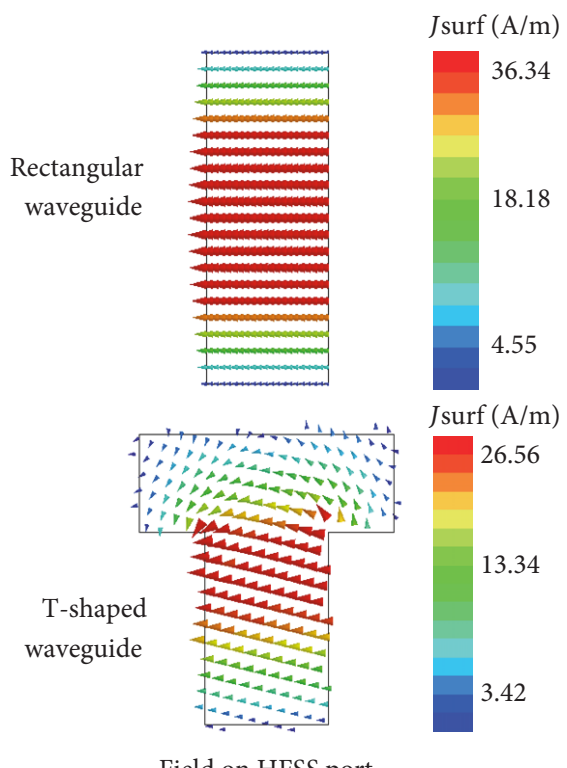

Field on HFSS port

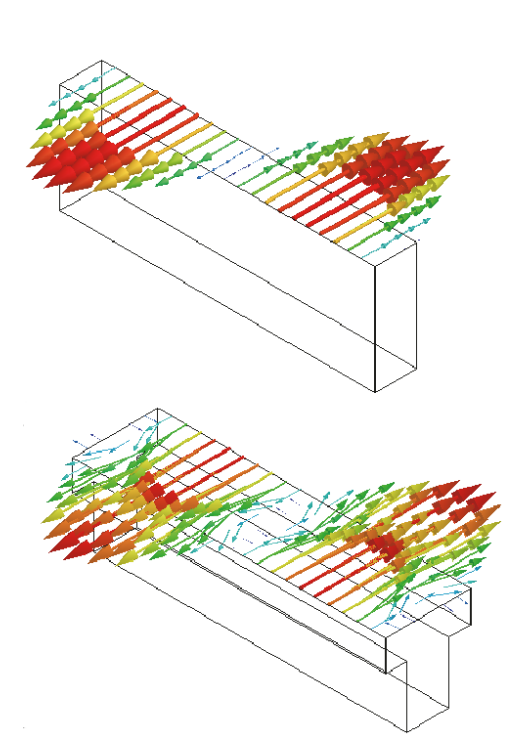

Surface current

(b)

Figure 1: Geometry and field distributions of the rectangular and T-shaped waveguide. (a) Configuration. (b) Port field in dominant mode and surface current on the top plate.

The operating center frequency of antenna array is $35 \mathrm{GHz}$ and the guide wavelength $\lambda_{\mathrm{g}}$ is set as $15.02 \mathrm{~mm}$. The width $b$ of the rectangular waveguide is set as $2 \mathrm{~mm}$ and the height $a=5.21 \mathrm{~mm}$ can be derived. For T-shaped waveguide, the dimensions are chosen as the following rules:

(a) The bottom width $b$ is the same as the rectangular one.

(b) The top width $a_{1}$ should be wide enough to support the resonant slot and the higher order modes should be avoided over the working band. The length of the resonant radiating slot is around $\lambda$, where $\lambda=$ $8.57 \mathrm{~mm}$ is the wavelength at the center frequency. Considering that the slot should be rotated at a certain angle, $a_{1}$ can be shorter than $\lambda / 2$.

(c) The heights $a_{1}$ and $a_{2}$ should be chosen to achieve the demand guide wavelength.

Finally, $b_{1}=4.1 \mathrm{~mm}, a_{1}=1.5 \mathrm{~mm}$, and $a_{2}=2.94 \mathrm{~mm}$ are decided. To reveal the operating mechanism of these dimensions, the parameter study is presented and the method is to sweep one parameter and fix the others. Figure 2 illustrates the propagation constant curves where $\beta_{\mathrm{R}}$ and $\beta_{\mathrm{T}}$ represent the propagation constant of rectangular waveguide and Tshaped waveguide and the subscripts 1 and 2 represent the dominant mode and second higher mode. It can be found that the parameter $b_{1}$ takes the main effect on the secondorder mode but a slight effect on dominant mode. Instead, the parameters $a_{1}$ and $a_{2}$ affect mostly the dominant mode. Compared with the rectangular waveguide, for the same $\beta$, the T-shaped waveguide shows a lower profile where $a_{1}+a_{2}<a$.

\section{Design of $2 \times 8$ Antenna Array}

3.1. Self-Admittance of Radiating Slot. As mentioned previously, the inclined radiating slots can be treated as the regular ones which can also be equivalent to shunt admittance in the resonant state. The inclined angle refers to the admittance and the length of the slot is used to achieve the resonance. The single slot model with period boundary condition (PBC) based on the T-shaped waveguide and normalized admittance curve are shown in Figure 3 where the thickness and the width of the slot are $0.5 \mathrm{~mm}$ and $0.6 \mathrm{~mm}$, respectively. The admittance extractions are carried out by using $S$-parameter results [8]. These obtained curves referring to the resonant 


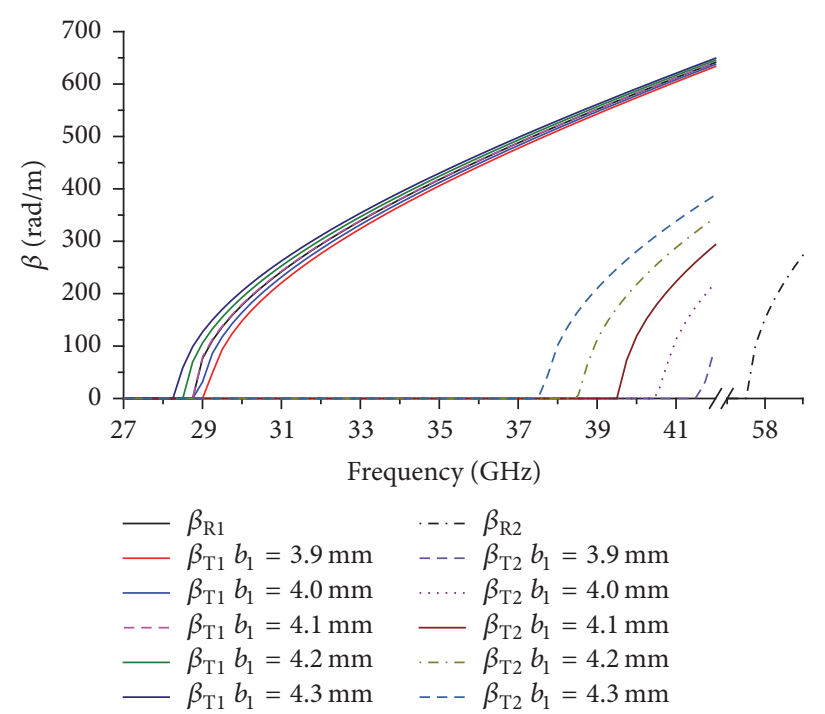

(a)

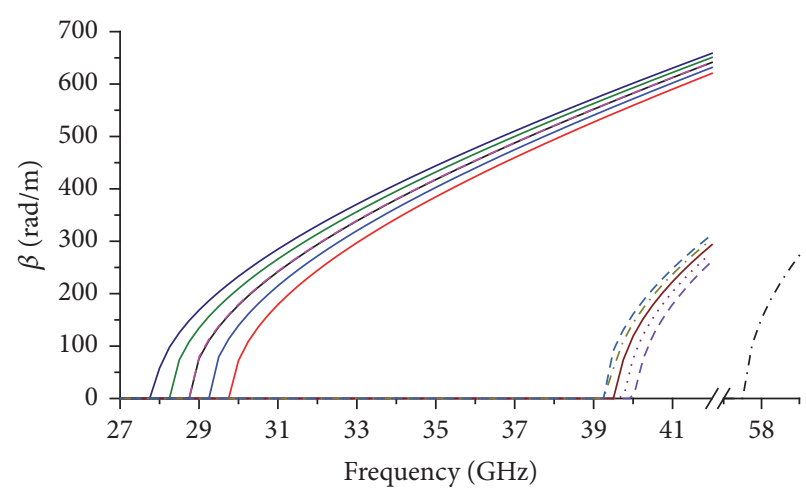

$$
\begin{array}{ll}
-\beta_{\mathrm{R} 1} & --\beta_{\mathrm{R} 2} \\
-\beta_{\mathrm{T} 1} a_{1}=1.3 \mathrm{~mm} & ---\beta_{\mathrm{T} 2} a_{1}=1.3 \mathrm{~mm} \\
-\beta_{\mathrm{T} 1} a_{1}=1.4 \mathrm{~mm} & \cdots \ldots \beta_{\mathrm{T} 2} a_{1}=1.4 \mathrm{~mm} \\
--\beta_{\mathrm{T} 1} a_{1}=1.5 \mathrm{~mm} & -\beta_{\mathrm{T} 2} a_{1}=1.5 \mathrm{~mm} \\
-\beta_{\mathrm{T} 1} a_{1}=1.6 \mathrm{~mm} & --\beta_{\mathrm{T} 2} a_{1}=1.6 \mathrm{~mm} \\
-\beta_{\mathrm{T} 1} a_{1}=1.7 \mathrm{~mm} & ---\beta_{\mathrm{T} 2} a_{1}=1.7 \mathrm{~mm}
\end{array}
$$

(b)

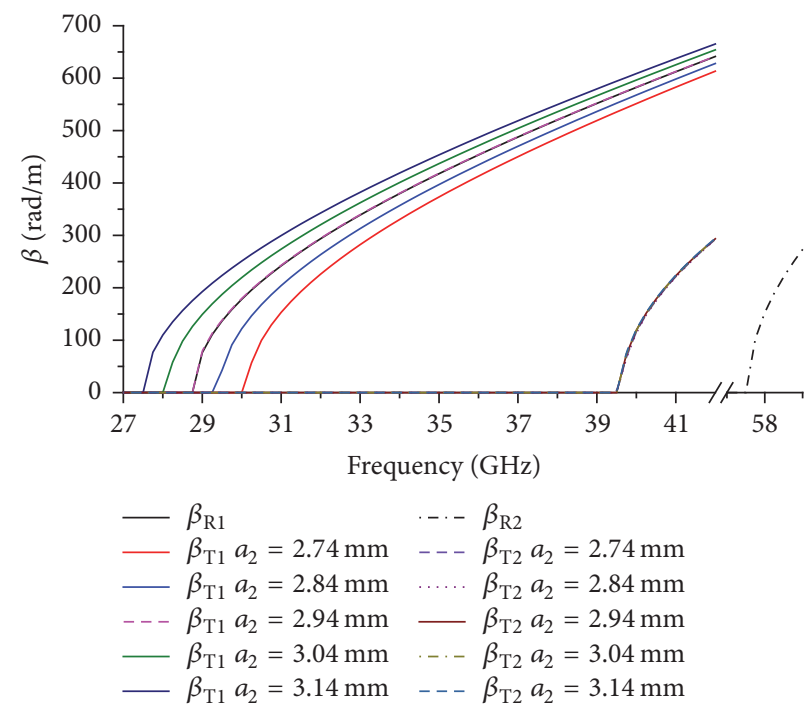

(c)

FIGURE 2: The propagation constant curves of rectangular waveguide and T-shaped waveguide versus frequency in dominant mode and second higher mode versus (a) $b_{1}$, (b) $a_{1}$, and (c) $a_{2}$.

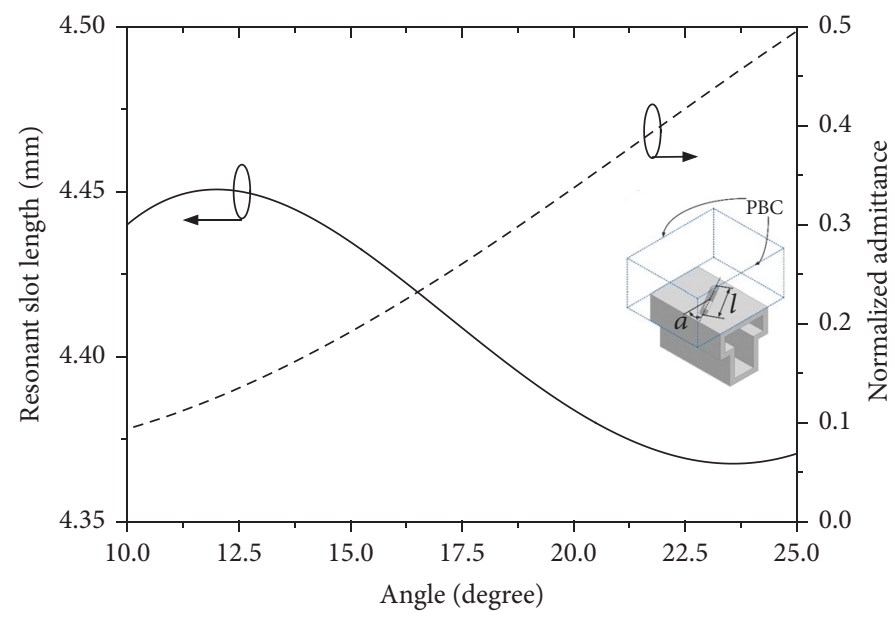

FIGURE 3: Normalized admittance and resonant length regarding the inclined angle. 


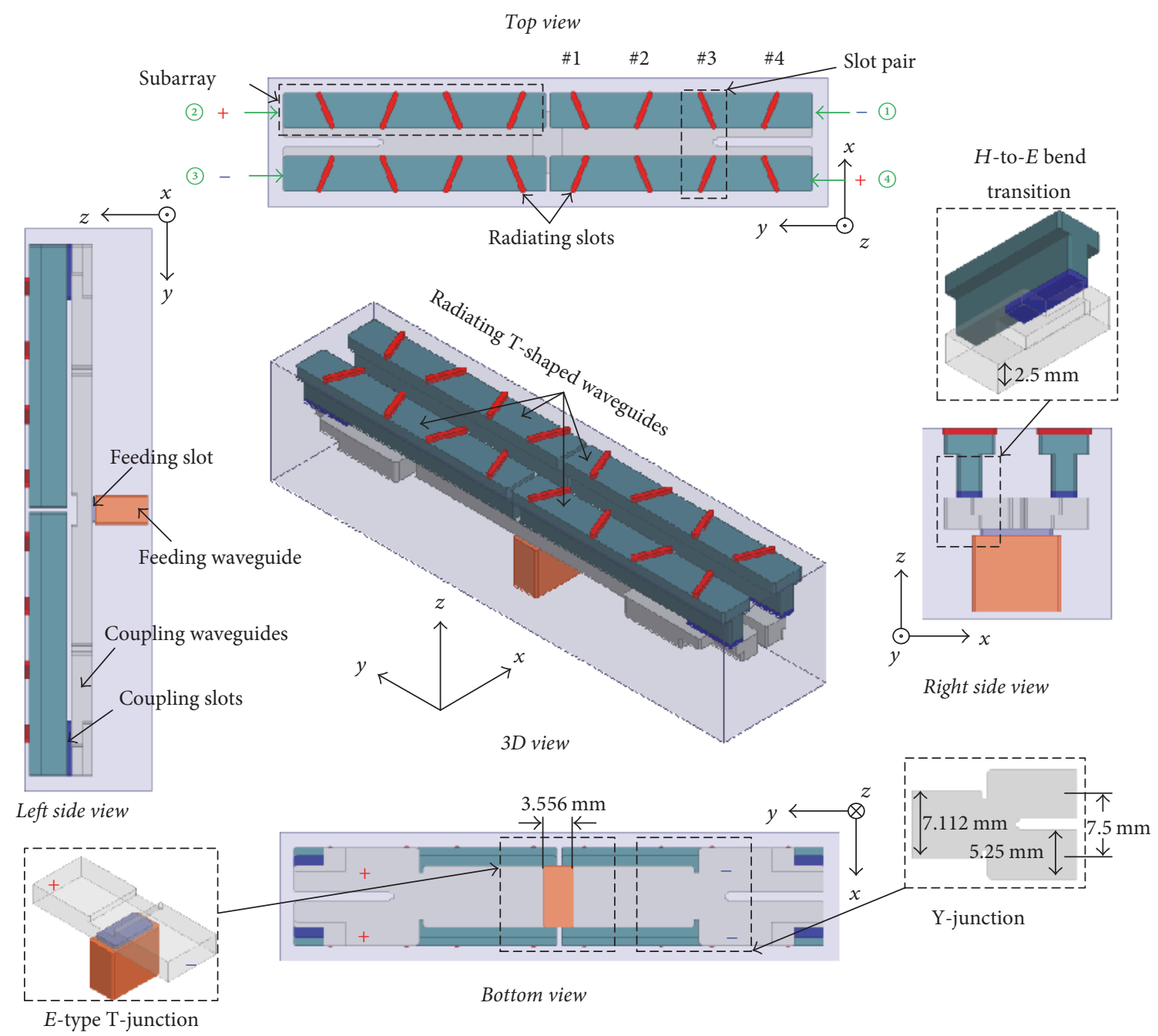

FIgURE 4: Structure of $2 \times 8$ antenna array.

admittance and the resonant length to angle variation are utilized to satisfy the input matching and the aperture field distributions. The PBC along waveguide direction is adopted for the purpose of accurate design which considers the mutual coupling between slots.

3.2. Antenna Array Structure. The slot pair consisting of two reverse inclined slots is introduced in the $2 \times 8$ antenna array to suppress the cross-polarization as depicted in Figure 4. Four subarrays are designed and fed from outside edge. The uniform distribution of aperture is desired and all slots have the same admittance of 0.25 . The slot length and the inclined angle can be evaluated from Figure 3 by an interpolation method. However, the actual boundary conditions of the slots are different owing to the mutual coupling from the adjacent slots, the effect of short end from radiating waveguide, and feeding port. Therefore, the dimensions of slots are optimized individually and the final dimensions of slots from 1 to 4 are as follows: slot length $=4.42,4.36,4.36$, and $4.43(\mathrm{~mm})$ and inclined angle $=17.6,18.6,20.35$, and 20.15 (degree). The radiation amplitude ratio and phase difference of the four slots are as follows: amplitude ratio is $1.01: 0.988: 0.988: 1$ and the phase difference is $0.79,-0.05,-0.24$, and -0.98 (degree). Obviously, the uniform distribution of aperture is realized.

Due to the reversed slots of slot pair, the radiating waveguides in subarray 1 and subarray 4 and subarray 2 and subarray 3 should be fed out-of-phase. The symbols + and - in figures denote out-of-phase condition. Two Y-junctions and an $E$-type T-junction are designed for equal power splitting with in-phase and out-of-phase condition. Four $\mathrm{H}$ to- $E$ bend transitions are invented to connect the radiating waveguides and the coupling waveguides. According to the mirror symmetry, the coupling slots in subarrays 1 and 4 are placed in opposite direction to achieve the out-of-phase condition. The subarrays 2 and 3 have the same property. Finally, the out-of-phase conditions between the adjacent radiating waveguides are obtained and all slots are fed inphase.

3.3. Simulation and Analysis. The simulated E-plane radiation patterns at the center frequency of antenna array are shown in Figure 5. As is known to all, the theoretical sidelobe 


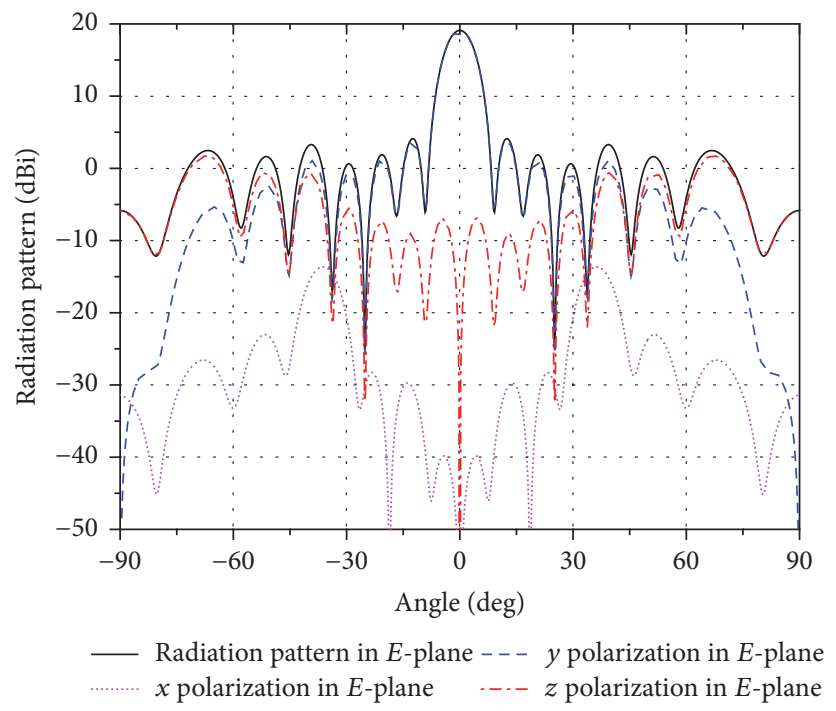

FIGURE 5: Simulated $E$-plane radiation patterns at $35 \mathrm{GHz}$.

level (SLL) of the uniform array is around $-13.2 \mathrm{~dB}$ and then tapered from the first sidelobe to far-out sidelobe. The simulated gain is $19.1 \mathrm{dBi}$ and the observed SLL is $-15 \mathrm{~dB}$ with almost uniform value over a wide angle range which is different from the theoretical situation.

To figure out the reason, the radiation pattern in $E$ plane is separated into $x, y$, and $z$ components as shown in Figure 5 which denote the corresponding polarization radiation pattern in $E$-plane. It can be seen that the $y$ component is copolarization and the others are cross-polarizations. The $x$ component is suppressed well by the slot pairs but the $z$ component is intense at the far-out sidelobe. The $z$ component is excited by radiating slots and then propagates as TM surface wave [11] to the edges of antenna array as depicted in Figure 6(a) which means the edge diffractions influence the radiation pattern.

To suppress the surface wave, the metal fences [12] are introduced between slots as shown in Figure 6(b). The height of fences is $f_{h}=\lambda / 4$ and the width of the fences is $f_{w}$ $=2 \mathrm{~mm}$. Owing to the effect of fences, the dimensions of radiating slots should be modified. The optimal values from slot 1 to slot 4 are as follows: slot length $=4.65,4.65,4.65$, and $4.57(\mathrm{~mm})$ and inclined angle $=20.5,20.5,20.5$, and 20.5 (degree). It can be found that the differences between slots are decreased compared with the original one which demonstrate the mutual coupling suppression by the metal fences. The radiation amplitude ratio and phase difference of slots from 1 to slot 4 are also calculated: the amplitude ratio is $1: 1: 1.05: 1.08$ and the phase difference is $0,-0.65,0.66$, and -5.38 . Therefore, the uniform aperture excitation is realized.

The $E$-field distribution in the $y-z$ plane is shown in Figure 6(c) for comparison. On the edge of the waveguide, the $E$-field of the original antenna is obviously stronger than the fenced one. As described previously, the edge diffractions may influence the radiation pattern and the power flow distribution is shown in Figure 6(d) for demonstration. The power flow direction on the edge of the original antenna is about $45^{\circ}$ which contributes to the far-out sidelobe. For the antenna with fences, most of the power flow is along the boresight.

To demonstrate the analysis of these structures, the radiation pattern of slotted waveguide antenna based on the regular waveguide and a T-shaped waveguide with/without fences are depicted in Figure 7. The spacings between slots of the three antenna arrays are all $7.5 \mathrm{~mm}$. For the regular waveguide, the width and height are $2 \mathrm{~mm}$ and $5.21 \mathrm{~mm}$, respectively, which can provide the same guide wavelength as the proposed one. It is clear that the gains of the proposed antenna arrays are much higher than the regular one due to the broad top wall which contributes larger aperture size. The first SLL of the antenna array with fences is $13.51 \mathrm{~dB}$ and the far-out sidelobe is well suppressed compared with the others. Furthermore, the fences between slots can correct the polarization and more energy is radiated in $y$-polarization which is benefit for the gain enhancement. The simulated gain is improved from the regular one of $16.22 \mathrm{dBi}$ to the proposed one of $19.1 \mathrm{dBi}$ and enhanced to $20.21 \mathrm{dBi}$ by the fences.

\section{Fabrication and Measurement Results}

In order to demonstrate our design, the proposed $2 \times 8$ antenna array was fabricated and assembled by screws and alignment pins as shown in Figure 8. The return loss was measured by using Agilent E8363C vector network analyzer. Figure 9 shows the simulated and measured return loss. The measured locus is in good agreement with the simulation. The simulated and measured impedance bandwidths with a return loss better than $15 \mathrm{~dB}$ are from $33.85 \mathrm{GHz}$ to $35.82 \mathrm{GHz}$ (5.63\%) and $33.96 \mathrm{GHz}$ to $36.29 \mathrm{GHz}(6.66 \%)$, respectively.

The simulated and measured radiation patterns at $35 \mathrm{GHz}$ are shown in Figure 10. The measured copolarization radiation patterns in $E$-plane and $H$-plane are in good agreement with the simulations. The measured cross-polarization levels in $E$-plane and $H$-plane are both less than $-25 \mathrm{~dB}$. 


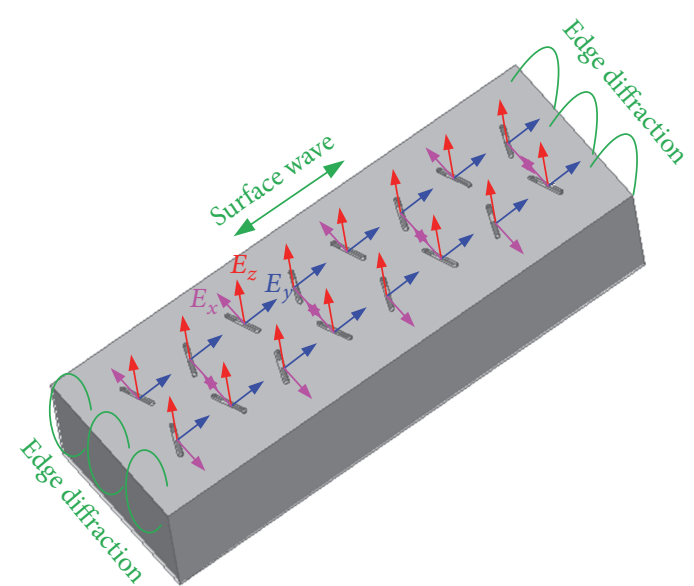

(a)

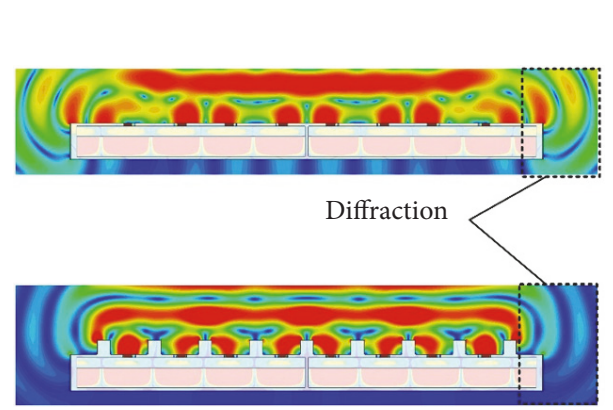

(c)

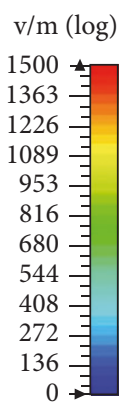

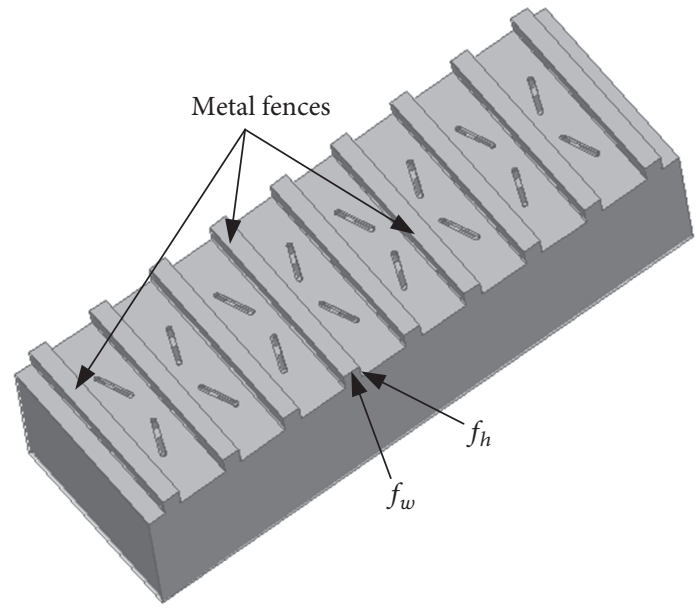

(b)

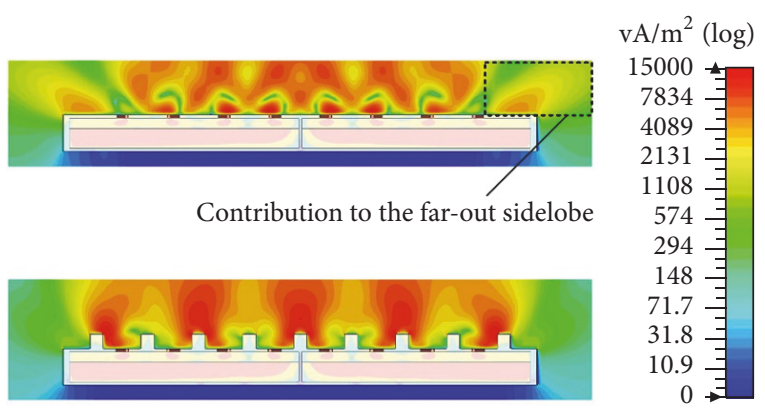

(d)

FIGURE 6: (a) Radiation analysis of antenna array. (b) Antenna array with metal fences. (c) E-field distribution. (d) Power flow distribution in the $y$ - $z$ plane at $35 \mathrm{GHz}$.

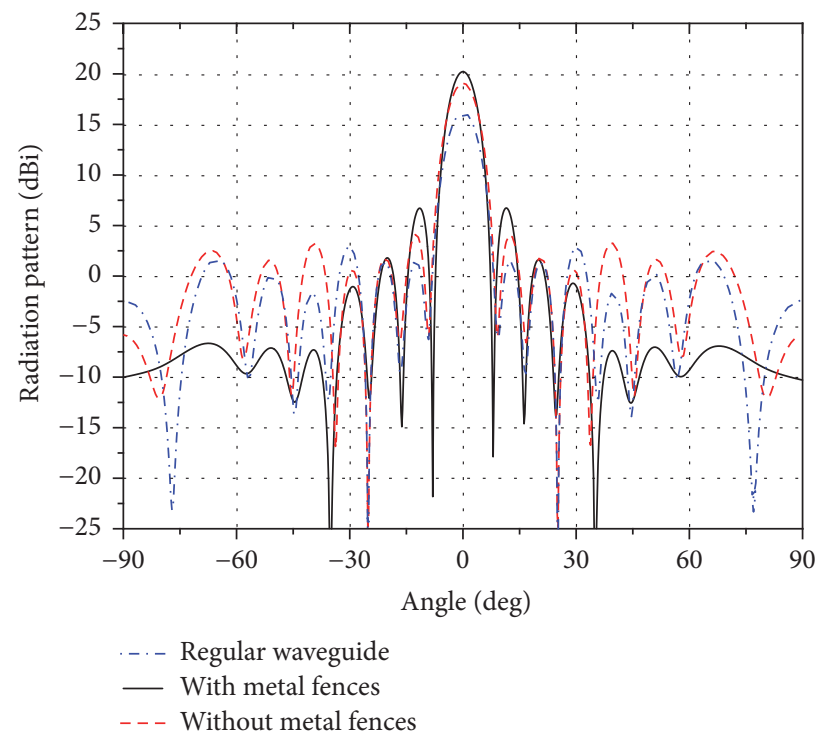

FIGURE 7: Radiation pattern of slot antenna based on the regular waveguide, the T-shaped waveguide with/without fences in $E$-plane at $35 \mathrm{GHz}$. 


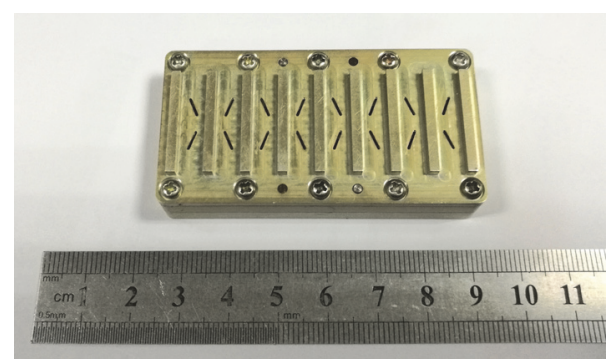

Figure 8: Photography of the fabricated $2 \times 8$ antenna array.

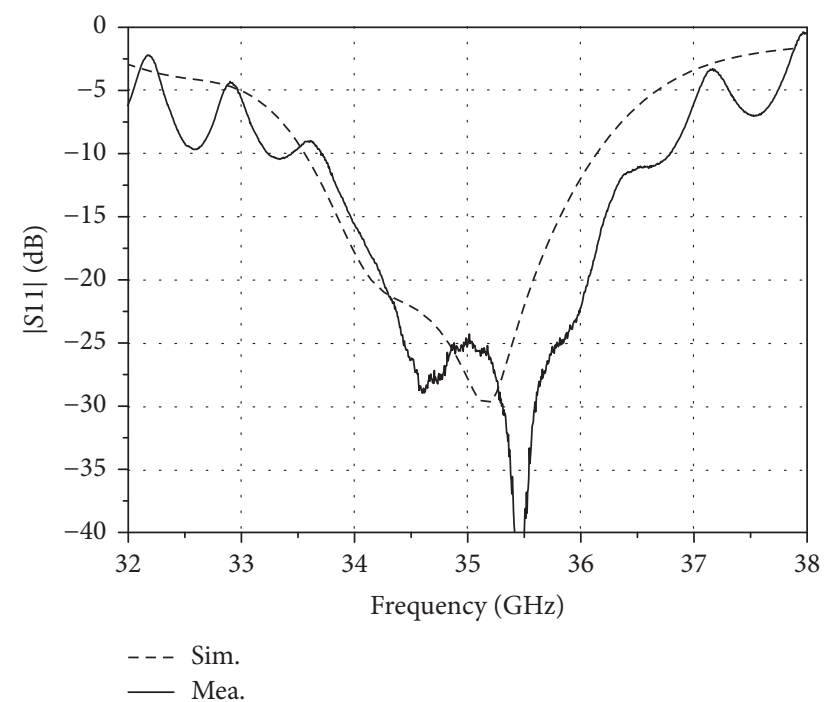

FIGURE 9: Simulated and measured return loss of $2 \times 8$ antenna array.

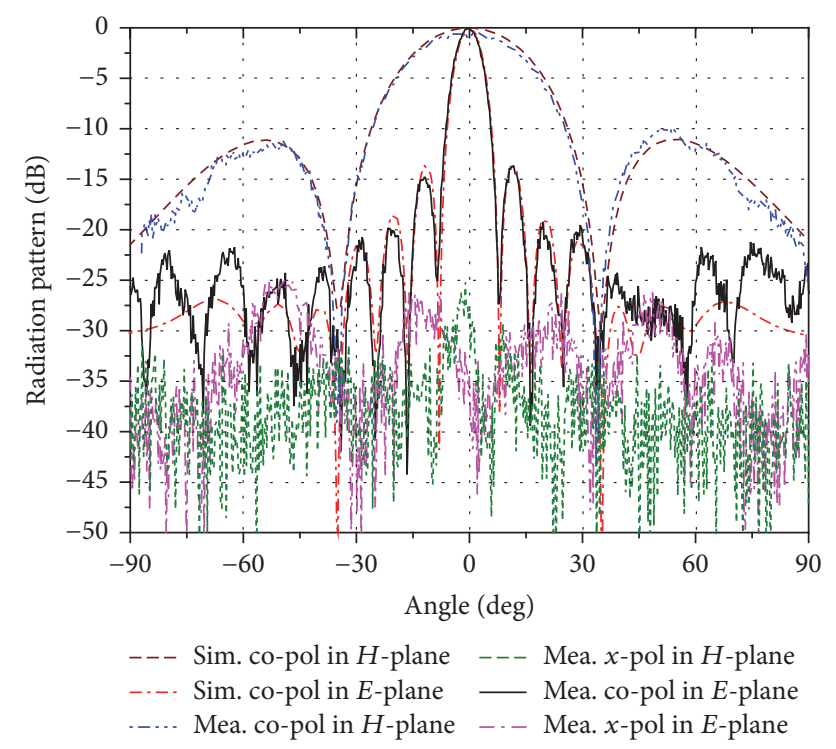

FIGURE 10: Simulated and measured normalized radiation pattern of the $2 \times 8$ antenna array at $35 \mathrm{GHz}$.

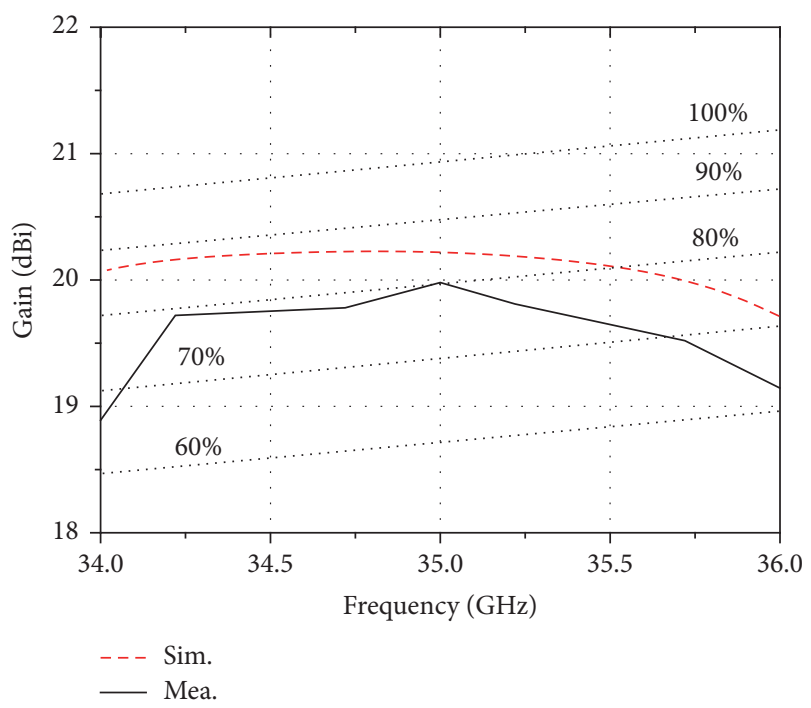

FIGURE 11: Gain and antenna efficiency of the proposed antenna array at $35 \mathrm{GHz}$.

The frequency characteristics of gain and antenna aperture efficiency are depicted in Figure 11. It can be found that the measured gains are a little less than the simulations owing to the conductor loss. The maximum gain of $19.98 \mathrm{dBi}$ is measured at $35 \mathrm{GHz}$ and the corresponding antenna aperture efficiency is $80.27 \%$.

\section{Conclusions}

A $2 \times 8$ antenna array based on T-shaped cross-section waveguide working at Ka-band is proposed. The operating mode of the waveguide is analyzed and designed to operate in dominant mode over the working band. Compared with the rectangular waveguide, the T-shaped waveguide has a lower profile and the broadened top plates can support the resonant radiating slots without cutting into the adjacent broad walls. To suppress the surface wave, the metal fences are introduced between slots and the radiation performance is improved. The measured results agree well with the simulations which validate our design. This novel structure has great potential application in communication and radar systems.

\section{Disclosure}

An earlier version of this work was presented as a conference paper at iWAT, 2015.

\section{Conflicts of Interest}

The authors declare that they have no conflicts of interest.

\section{Acknowledgments}

This work was supported by China Postdoctoral Science Foundation Funded Project under Grant 2016M591740. 


\section{References}

[1] A. Traille, J. Ratner, G. D. Hopkins, and V. Tripp, "Development of a novel faceted, conformal, slotted-waveguide subarray for sensor applications with full $360^{\circ}$ azimuth tracking capabilities," in Proceedings of the 2007 IEEE Antennas and Propagation Society International Symposium (ISAP' 07), pp. 3828-3831, Honolulu, HI, USA, June 2007.

[2] D. Dogan and Ö. A. Civi, "A novel edge wall waveguide slot antenna," in Proceedings of the 4th IEEE International Symposium on Phased Array Systems and Technology (ARRAY' 10), pp. 453-456, Waltham, MA, USA, October 2010.

[3] M. Moradian, "Untilted edge-slot antenna arrays fed by wiggly double-ridged waveguide," Electronics Letters, vol. 51, no. 24, pp. 1966-1967, 2015.

[4] D. Gray, X. Xin, Y. Zhu, and J. Le Kernec, "Structural slotted waveguide antennas for multirotor UAV radio altimeter," in Proceedings of the 2014 IEEE International Conference on Signal Processing, Communications and Computing (ICSPCC' 2014), pp. 819-824, Guilin, China, August 2014.

[5] H. Watanabe, T. Ohba, S. Yamaguchi et al., "Waveguide edgeshunt slot array antenna with Z-shaped slot partially overlapping the waveguide wall," in Proceedings of the Microwave Conference (APMC '14), 2014 Asia-Pacific, pp. 723-725, Sendai, Japan, November 2014.

[6] S. Clauzier, S. Avrillon, L. Le Coq, M. Himdi, F. Colombel, and E. Rochefort, "Slotted waveguide antenna with a near-field focused beam in one plane," IET Microwaves, Antennas and Propagation, vol. 9, no. 7, pp. 634-639, 2015.

[7] W. Wang, J. Jin, J.-G. Lu, and S.-S. Zhong, "Waveguide slotted antenna array with broadband, dual-polarization and low crosspolarization for X-band SAR applications," in Proceedings of the 2005 IEEE International Radar Conference Record (RADAR' 2005), pp. 653-656, Arlington, VA, USA, May 2005.

[8] T. Li, H. Meng, and W. Dou, "Design and implementation of dual-frequency dual-polarization slotted waveguide antenna array for Ka-band application," IEEE Antennas and Wireless Propagation Letters, vol. 13, pp. 1317-1320, 2014.

[9] P. Magnusson, P. Dimming, C. Lin, and A. Ostergaard, "A thermally stable dual-polarized waveguide array," in Proceedings of the 9th European Conference on Antennas and Propagation (EuCAP’ 15), pp. 1-5, Lisbon, Portugal, April 2015.

[10] T. Li and W. Dou, "Design of a new waveguide slotted antenna array," in Proceedings of the International Workshop on Antenna Technology (iWAT'2015), pp. 211-213, Seoul, South Korea, March 2015.

[11] R. E. Collin, Field Theory of Guided Waves, IEEE Press, 1991.

[12] Y. Xiaole, N. Daning, L. Shaodong, L. Zhengjun, and W. Wutu, "Design of a wideband waveguide slot array antenna and its decoupling method for synthetic aperture radar," in Proceedings of the 38th European Microwave Conference (EuMC' 2008), pp. 135-138, Amsterdam, The Netherlands, October 2008. 


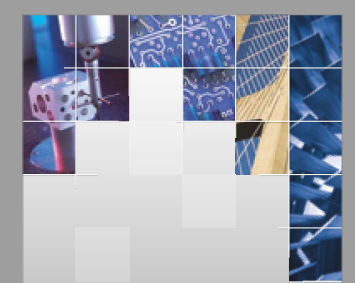

\section{Enfincering}
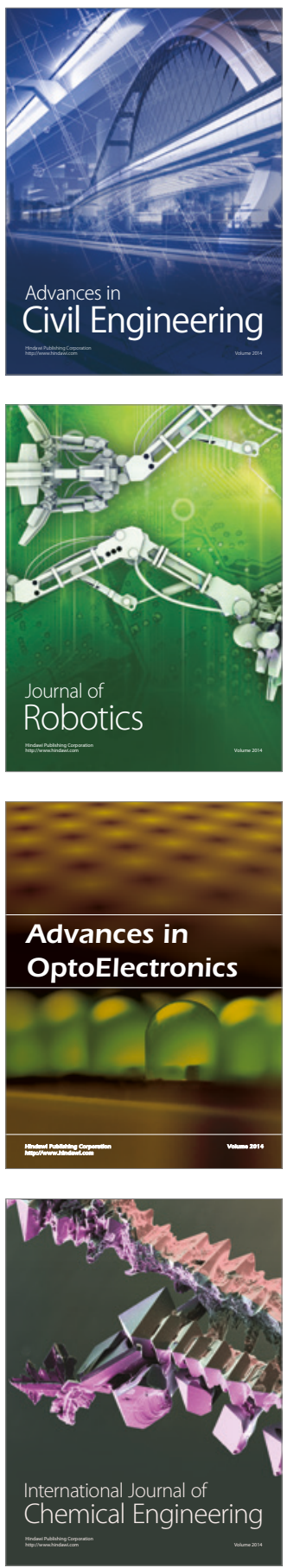

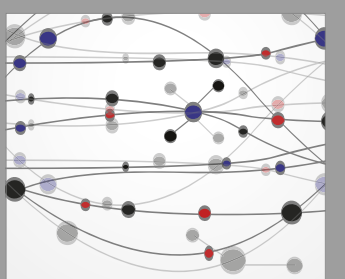

The Scientific World Journal

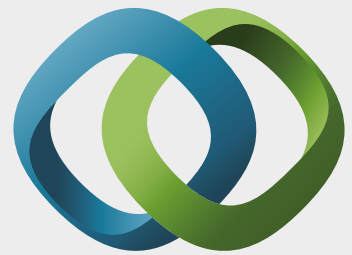

\section{Hindawi}

Submit your manuscripts at

https://www.hindawi.com
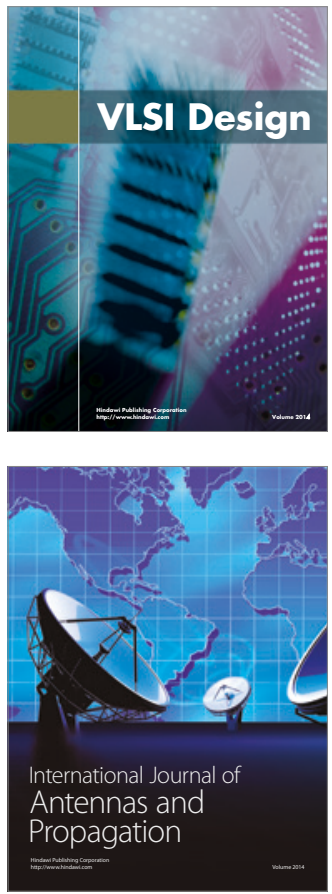

\section{Rotating}

Machinery
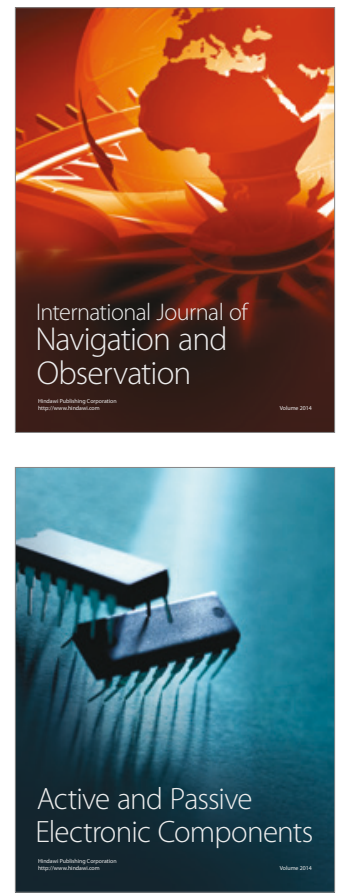
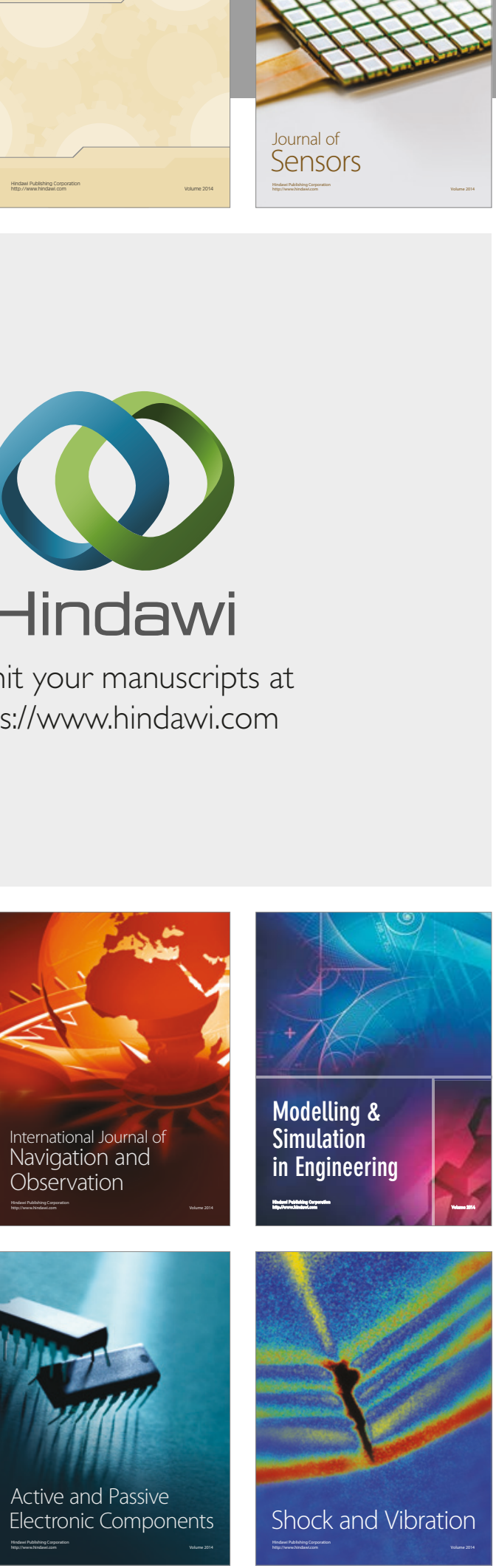
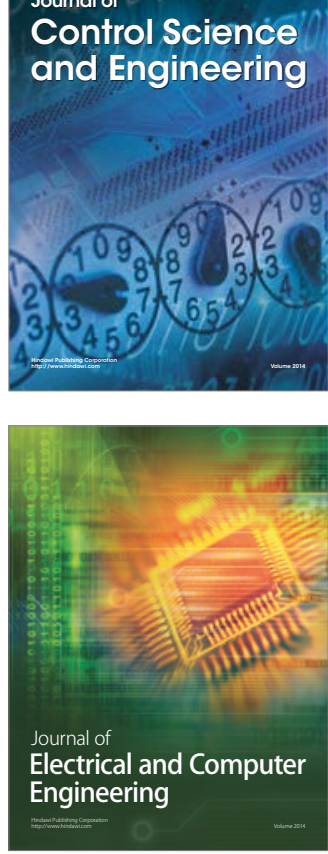

Distributed

Journal of

Control Science

and Engineering
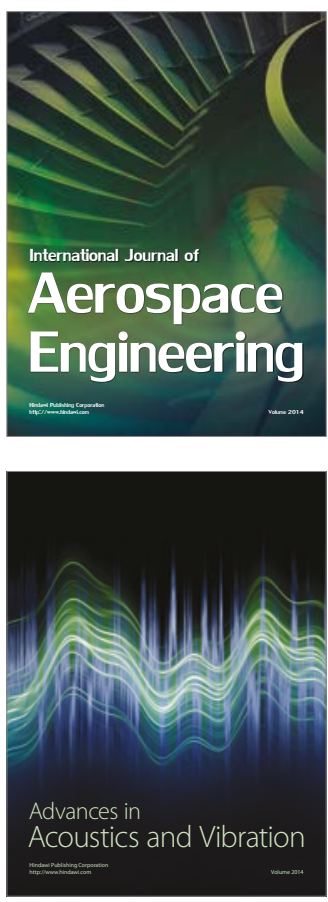

Sensor Networks 